\title{
Über die Reduktion von Zwangsmaßnahmen durch eine „offene Türpolitik“
}

\author{
About the Reduction of Compulsory Measures by an „Open Door Policy“
}

Autoren

Institute
Undine E. Lang ${ }^{1}$, Marc Walter ${ }^{1}$, Stefan Borgwardt ${ }^{1}$, Andreas Heinz ${ }^{2}$

${ }^{1}$ Universitäre Psychiatrische Kliniken Basel (UPK), Basel/Schweiz

${ }^{2}$ Campus Mitte, Charité - Universitätsmedizin Berlin

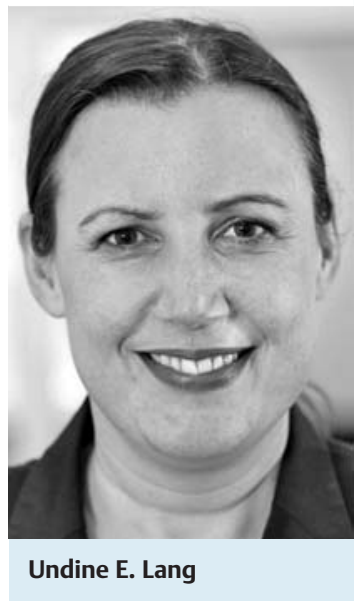

Bibliografie

Dol http://dx.doi.org/ 10.1055/s-0042-111032 Psychiat Prax 2016; 43: 299-301

(c) Georg Thieme Verlag KG Stuttgart · New York ISSN 0303-4259

Korrespondenzadresse Prof. Dr. med. Undine E. Lang Klinikdirektorin der Erwachsenen-Psychiatrischen Klinik EPK, Ordinariat Psychiatrie der Universität Basel Wilhelm-Klein-Straße 37 4056 Basel, Schweiz undine.lang@upkbs.ch
Zwangsunterbringungen gefährden die therapeutische Beziehung, Compliance sowie das Image der Psychiatrie und können mit Traumatisierung, Stigmatisierung und Folgestörungen von Patienten verbunden sein. Sie sind deshalb auf das unmittelbar zur Abwendung krankheitsbedingter Selbst- und Fremdgefährdung notwendige Maß zu reduzieren. In einer Zeit, in der annähernd 50\% der Allgemeinbevölkerung unter psychiatrischen Erkrankungen leidet, scheint es wichtig für die psychiatrische Versorgung, einzelne gefährdete Patienten zu erkennen und ihnen Sicherheit und Therapie zukommen zu lassen und gleichzeitig nicht die Masse der Patienten aus den Augen zu verlieren, die durch ebendiese Sicherheitsmaßnahmen, wenn sie zu pauschal angewendet werden, vor einer Behandlung in der Psychiatrie zurückschreckt.

Es gibt keine randomisierten kontrollierten Studien über den Nutzen und Schaden von der jeweiligen Unterbringungspraxis, Gesetzgebung sowie durchgeführten Zwangsmaßnahmen [1], entsprechend variiert deren Anwendung zwischen Stationen, Behandlungsteams und Ländern erheblich $[2,3]$. Im europaweiten Vergleich trat in Österreich die Mehrzahl der gerichtlich untergebrachten Patienten auf offenen Stationen ein, in der Slowakei wurde die Mehrzahl der freiwillig eintretenden Patienten auf geschlossenen Stationen behandelt [4]. Die Behandlungspraxis bzw. der Freiheitsgrad der Patienten ist mit dem gerichtlichen Unterbringungsstatus nicht zwangsläufig konform [4]. Dass eine „offene Türpolitik“ in der Psychiatrie Zwangsmaßnahmen reduzieren kann, ist in den Leitlinien der DGPPN und einem Statement der zentralen Ethikkommission der Bundesärztekammer formuliert worden und mehrfach beschrieben [5-10]. In einer aktuellen Arbeit zeigt sich bei ca. 350000 untersuchten Fällen, dass Komplikationen wie Suizide, Entweichungen und Gewalt in offen geführten psychiatrischen Versorgungskliniken nicht häufiger vorkommen, d.h., dass diese durch Türschließungen nicht verhindert werden können [11]. In den offenen Kliniken scheint jedoch mit weniger Zwangsmaßnahmen ein höherer Anteil von Patienten medikamentös erreicht und behandelt zu werden [11]. Allgemein findet sich auf geschlossenen im Vergleich zu offenen Stationen häufiger ein hohes Aggressionsniveau, mehr Zwangsmaßnahmen, ein bedrohliches Stationsklima, rigide Stationsregeln, weniger Mitsprache über das Behandlungsprozedere, ein geringer Einbezug der Patienten, eine geringe Compliance, ein geringes therapeutisches (v.a. psychotherapeutisches) Angebot und eine geringe emotionale Ansprechbarkeit der Bezugspersonen $[7,8,10,12]$.

Diese qualitativen Behandlungsunterschiede, die sich auf Zwangsmaßnahmen auswirken, lassen sich in einigen Untersuchungen auf ein anderes Klientel der behandelten Patienten zurückführen und können nicht alleine auf den mechanischen Zustand der geöffneten oder geschlossenen Tür zurückgeführt werden, bzw. auf die Situation, dass geschlossene Türen durch personelle Präsenz im Eingangsbereich ersetzt werden. Komplexe Mechanismen führen aber zu einer veränderten Therapieatmosphäre wenn Stationen geschlossen sind [10]. Die Verbindung aus geöffneter Tür und Reduktion von Zwang wird entsprechend kaum durch randomisiert-kontrolliert verblindetes Öffnen und Schließen der Eingangstür von psychiatrischen Kliniken $\mathrm{zu}$ untersuchen sein, weil verschiedenste Behandlungsaspekte und Mechanismen geändert werden müssen, um überhaupt Türen verantwortungsvoll in einer Klinik mit Regelversorgung öffnen zu können. Diese Veränderungen haben dann wiederum Auswirkungen auf die Häufigkeit von Zwangsmaßnahmen. In diesem Editorial möchten wir versuchen, einige dieser Aspekte zu beleuchten, wie offene Türen in der Regelversorgung indirekt Zwang, qualitative Behandlungsaspekte und atmosphärische Aspekte beeinflussen könnten. Ausgehend 
von Erfahrungen an der Berliner Charité wurde ein Projekt der Türöffnung auch in Basel initiiert [10]. In den Universitären Kliniken in Basel (UPK) wurden Patienten initial (2012) auf 4 geschlossenen Stationen (2 Stationen mit Suchtschwerpunkt, 2 Stationen Allgemeinpsychiatrie) aufgenommen, 6 Stationen waren durchgehend geöffnet und übernahmen oder verlegten Patienten von/ auf die geschlossenen Stationen. Ausgenommen von dem Öffnungsprojekt war die Alterspsychiatrie (2 Stationen), die geschlossen blieb und die Privatklinik (2 Stationen), die immer offen war [9, 10]. Bis 2012/2013 wurden 2 der 4 Stationen geöffnet, 2 weitere öffnen seit 2014/2015 fakultativ mit einem steigenden, hohen Anteil von ca. 80-90\% der Zeit.

\section{Die Konzentration von Akutpatienten und Crowding erhöht Zwangsmaßnahmen \\ $\nabla$}

Die wichtigste Voraussetzung für die Öffnung einer Klinik ist die Verteilung von Akutpatienten, damit der Kontakt mit den einzelnen Patienten, die neu eintreten und/oder eigen- und/oder fremdgefährdend sind, intensiviert werden kann. Steigt die Konzentration von Neuaufnahmen und untergebrachten Patienten dagegen auf einer Station an, reduziert sich indirekt der Personalschlüssel und Kontakte sind nicht mehr engmaschig möglich.

In den UPK Basel wurde im Zuge der Öffnung der 4 ehemals geschlossenen Stationen korrespondierend die Liegedauer dieser Akutstationen verlängert und die Aufnahme von Patienten erfolgte vorrangig auf den bestehenden 6 offenen Stationen. Entsprechend wurden Überbelegungen auf den ehemals geschlossenen Stationen abgebaut, die Verlegungen (beispielsweise wegen Suizidalität oder um auf der Aufnahmestation Platz zu schaffen) wurden gestoppt, und damit die Patienten so verteilt, dass es nicht zu einem Crowding von Schwerkranken kommen konnte. Faktoren wie eine niedrige Bettenmessziffer, die Überbelegung psychiatrischer Stationen oder eine Konzentration von Akutpatienten auf einer Station erhöhen die Zahl gewalttätiger Übergriffe und erfordern in der Folge vermehrt Zwangsmaßnahmen [6, 7, 13 -15]. In einem Review über Prädiktoren von Gewalt und korrespondierenden Zwangsmaßnahmen war Crowding einer der 3 wichtigsten Faktoren, der Aggressionen und damit konsekutiv Zwangsmaßnahmen auslöst [14, 15]. Ein „Umschiffen“ der geschlossenen Akutstation durch eine offene Kriseninterventionsstation kann entsprechend zu einer Reduktion von Zwangsmaßnahmen und weiteren Komplikationen führen [16].

\section{Einbezug von Patienten in die Therapie senken Zwangsmaßnahmen}

Im Rahmen einer offenen Türpolitik können Patienten nicht automatisch ungefragt auf eine „sichere“ geschlossene Station aufgenommen werden, stattdessen ist ein intensives Assessment durch die Behandler erforderlich, die genau beurteilen müssen, ob jemand eine personelle Intensivbetreuung braucht und/oder wegen des Patienten die Türe geschlossen werden muss. Bei Eintritt wird mit dem Patienten verhandelt, unter welchen Umständen dieser bereit ist, auf einer Station zu bleiben. Dies bedeutet wiederum einen stärkeren Einbezug von Patienten, eine höhere Flexibilität bezüglich der individuellen Bedürfnisse des Patienten und eine bessere Information und Aufklärung über die Diagnose und Möglichkeiten der Behandlung. Auf der Station selbst werden alle Patienten in die Entscheidung involviert, ob die Türe ge- schlossen werden muss und es wird engmaschig evaluiert, ob der Zustand der Patienten sich verändert hat, und geöffnet werden kann oder geschlossen werden muss. In Basel und Berlin wurde parallel zum Öffnungsprozess ein Behandlungsbeirat gegründet und eine Patientenverfügung bzw. Behandlungsvereinbarung in Kooperation mit anderen Kliniken entwickelt. Zwangsmaßnahmen können reduziert werden durch bestehende Zielvereinbarungen, Patientenorientierung, Bezugspflege, „Shared Decision Making“, Behandlungsvereinbarungen, Flexibilität in der Therapie und Angebote zur Wahrnehmung unabhängiger Beschwerdeinstanzen sowie Transparenz und Information $[6,13,14,17,18]$.

\section{Therapeutische Angebote senken Zwangsmaßnahmen $\nabla$}

Durch das Öffnungskonzept in Basel entwickelten alle „Akutaufnahmestationen“ sukzessive diagnosespezifische Konzepte. Damit erhalten die Patienten mehr Angebot, Information und Wahlmöglichkeiten in ihrem Behandlungspfad und gelangen direkt an den Experten und einem ihrer Diagnose zugeschnittenem Konzept. Die Psychotherapieangebote wurden auf den neu geöffneten Stationen je nach neuem diagnostischem Schwerpunkt ausgebaut und sind für jeden eintretenden Akutpatienten zugänglich. Die Stationsatmosphäre hat sich auf den neu geöffneten Stationen verbessert, da die Aufnahmeteams, die ursprünglich auf alle möglichen Situationen reagieren mussten, Zeit gewinnen konnten, ein diagnosespezifisches Konzept und aktiv individualisierte Lösungen im Umgang mit Krisensituationen zu erarbeiten [9]. Durch Ausbau der therapeutischen Angebote wurden Zwangsmaßnahmen verringert und die Selbstwirksamkeit der Patienten kann erhöht werden $[8,9]$.

\section{Eine gute Stationsatmosphäre senkt Zwangs- maßnahmen \\ $\nabla$}

Das Sicherheitserleben bei den Pflegeteams der neu geöffneten Stationen stieg an, da es weniger Übergriffe gab und entsprechend signifikant weniger Zwangsmaßnahmen [8, 9]. Auch wurde durch die Erhöhung der Liegedauer auf den ehemaligen Aufnahmestationen möglich, dass die Teams „Besserung“ und „Remission“ erleben, woraus eine ressourcenorientiertere Haltung resultiert. Damit steigen im Rahmen eines Öffnungskonzepts sowohl die Patientenzufriedenheit, die Stationsatmosphäre, die Behandlungsqualität als auch der Betreuungsschlüssel für Akutpatienten. Entsprechend sanken neben den Zwangsmaßnahmen auch die Zahlen der Patienten, die gegen ärztlichen Rat die Klinik verließen $[8,9]$.

Zwangsmaßnahmen sinken demnach, wenn eine gute Stationsatmosphäre besteht, der Umgang mit den Patienten respektvoll ist sowie eine wertschätzende und nicht regelorientierte individualisierte Haltung beim Team besteht, Patienten positiv gewürdigt werden, eine Wertlegung auf Prävention besteht, Reflexionsfähigkeit des Teams vorliegt und den Patienten so viel Kontrolle wie möglich erhalten bleibt $[6,8,13,14,17,18]$. 


\section{Erhalt der Privatsphäre und Bewegungsspielraum senken Zwangsmaßnahmen \\ $\nabla$}

Mit der Öffnung von Stationen gehen Reduktionen von Sicherheitsmaßnahmen einher, die sich relativieren, was wiederum zu einem weniger restriktiven Behandlungsklima beiträgt. So erübrigen sich Maßnahmen, die in die Privatsphäre von Patienten eingreifen wie zum Beispiel Durchsuchungen, Ausgangssperren, Entzug von Handy, Zigaretten, Nahrungsmitteln (Küche verschlossen, kein Gang zum Kiosk möglich), dem Ausschluss von extern stattfindenden Therapieprogrammen wie Sport, Ergotherapie etc. und es können alle Räumlichkeiten der Stationen wie Fitnessräume, Fernsehräume, Küche etc. durchgehend geöffnet werden. Dies wiederum erhöht den Platz pro Patient, zusätzlich zu dem Faktor, dass mehr Patienten sich im Ausgang befinden. Zwangsmaßnahmen sinken zudem, wenn die Intimsphäre gewahrt wird, Normalität auf einer Station vorherrscht sowie ein liberales Klima herrscht $[6,8,13,14,17,18]$. Die bestehende Privatsphäre, empfundene Autonomie und der Platz pro Patient auf einer Station steuern die Häufigkeit von Zwangsmaßnahmen genauso wie die Anzahl der abschließbaren Räume [15]. In einem aktuellen systematischen Review von 71 Studien zeigt sich, dass der stärkste Prädiktor von aggressivem Patientenverhalten mit 39\% der Fälle die Interaktion von Team und Patient und zwar eine Restriktion bzw. Verweigerung eines Patientenwunsches war [17].

\section{Teamreflexion und Nahbarkeit senkt Zwangs- maßnahmen \\ $\nabla$}

Ein Öffnungsprozess einer Station bedingt ein starkes Umdenken des Teams, das flexibler und nahbarer werden muss. In Basel war auf den geschlossenen Stationen mit den höchsten Zwangsmaßnahmen über Jahre keine Teamsupervision erfolgt. Diese musste mit der Öffnung eingeführt werden und stieß initial auf Widerstand. Auffällig war, dass mit der Öffnung der Stationstüren der Akutstationen auch die Diensträume geöffnet wurden, was zu einer besseren Ansprechbarkeit der Teams durch die Patienten führte. Im Auftreten von Zwangsmaßnahmen spielen Nahbarkeit und Selbstreflexion des Teams und die Auffassung über den Ursprung der Aggression eines Patienten eine wichtige Rolle [6, 8, $13,14,17,18]$. Aggression kann als internales am ehesten biologisch bedingtes Korrelat einer Erkrankung gesehen werden, dem dann durch eine (Zwangs-)Medikation kausal begegnet wird. Diese Interpretation des Verhaltens entlastet zwar Patienten vor Verantwortung, kann aber weniger Empathie seitens des Therapeuten bewirken [19]. Wird Aggression dagegen als externale Reaktion gesehen, liefern Interaktionen mit dem Team, Restriktionen, Frustrationen eine wesentliche Erklärung für das Verhalten. Diese kontextabhängige Interpretation von Aggression erfordert mehr Empathie und wird Reflexions- und Veränderungsbedarf in der Art der Behandlung und der Team-Patient-Beziehung erzeugen. Patientencharakteristika spielen eine kleine Rolle bei dem Einsatz von Zwangsmaßnahmen [17], die zum größten An- teil von interpersonellen Faktoren und von der Haltung des Behandlungsteams abhängen. Wird die Haltung und Situationen, in denen Zwang erforderlich war, reflektiert, finden entsprechend weniger Zwangsmaßnahmen statt, genauso wie „Nahbarkeit“ Zwangsmaßnahmen reduziert [6, 8, 13, 14, 17, 18].

\section{Literatur}

1 Muralidharan S, Fenton M. Containment strategies for people with serious mental illness. Cochrane Database Syst Rev 2006; 3: CD002084

2 Husum TL, Bjørngaard JH, Finset A et al. A cross-sectional prospective study of seclusion, restraint and involuntary medication in acute psychiatric wards: patient, staff and ward characteristics. BMC Health Serv Res 2010; 10: 89

3 Martin V, Bernhardsgrütter $R$, Göbel $R$ et al. The use of mechanical restraint and seclusion: comparing the clinical practice in Germany and Switzerland. Psychiat Prax 2007; 34 (Suppl. 02): S212 - 217

4 Rittmannsberger $H$, Sartorius $N$, Brad $M$ et al. Changing aspects of psychiatric inpatient treatment. A census investigation in five European countries. Eur Psychiatry 2004; 19: 483-488

5 Wiesing U. Stellungnahme der Zentralen Kommission zur Wahrung ethischer Grundsätze in der Medizin und ihren Grenzgebieten (Zentrale Ethikkommission) bei der Bundesärztekammer Zwangsbehandlung bei psychischen Erkrankungen. Dtsch Arztebl 2013; 110: 1334 1338

6 Gaebel W, Falkai P, Hrsg. Therapeutische Maßnahmen bei aggressivem Verhalten in der Psychiatrie und Psychotherapie. Reihe: S2 Praxisleitlinien in Psychiatrie und Psychotherapie. Heidelberg: Springer; 2007

7 Sollberger D, Lang UE. Psychiatry with open doors. Part 2: Therapeutic challenges. Nervenarzt 2014; 85: 319-325

8 Blaesi S, Gairing SK, Walter M et al. Safety, therapeutic hold, and patient's cohesion on closed, recently opened, and open psychiatric wards. Psychiat Prax 2015; 42: 76-81

9 Jungfer HA, Schneeberger AR, Borgwardt $S$ et al. Reduction of seclusion on a hospital-wide level: successful implementation of a less restrictive policy. J Psychiatr Res 2014; 54: $94-99$

10 Lang UE, Hartmann S, Schulz-Hartmann S et al. Do locked doors in psychiatric hospitals prevent patients from absconding? Eur J Psychiatry 2010; 24: 199-204

11 Huber CG, Schneeberger AR, Kowalinski E et al. Suicide Risk and Absconding in Psychiatric Hospitals with and without Open Door Policies: A 15-year Naturalistic Observational Study. Lancet Psychiatry [im Druck]

12 Sollberger $D$, Lang UE. Psychiatry with open doors. Part 1 : Rational for an open door for acute psychiatry. Nervenarzt 2014; 85: 312-318

13 Bak J, Brandt-Christensen M, Sestoft DM et al. Mechanical restraint which interventions prevent episodes of mechanical restraint? - a systematic review. Perspect Psychiatr Care 2012; 48: 83-94

14 Bak J, Zoffmann V, Sestoft DM et al. Mechanical Restraint in Psychiatry: Preventive Factors in Theory and Practice. A Danish-Norwegian Association Study. Perspect Psychiatr Care 2014; 50: 155-166

15 van der Schaaf PS, Dusseldorp E, Keuning FM et al. Impact of the physical environment of psychiatric wards on the use of seclusion. Br J Psychiatry 2013; 202: $142-149$

16 Steinert T, Eisele $F$, Goeser $U$ et al. Successful interventions on an organisational level to reduce violence and coercive interventions in in-patients with adjustment disorders and personality disorders. Clin Pract Epidemiol Ment Health 2008; 4: 27

17 Boumans CE, Egger JI, Souren PM et al. Nurses' decision on seclusion: patient characteristics, contextual factors and reflexivity in teams. J Psychiatr Ment Health Nurs 2012; 19: 264-270

18 Papadopoulos C, Ross J, Stewart D et al. The antecedents of violence and aggression within psychiatric in-patient settings. Acta Psychiatr Scand 2012; 125: 425-439

19 Lebowitz MS, Ahn WK. Effects of biological explanations for mental disorders on clinicians' empathy. PNAS 2014; 111: 17786 - 17790 